\title{
ON THE SITUATION OF NODES OF PLANE CURVES
}

\section{ROBERT TREGER}

1. Introduction. We consider complex plane algebraic curves with nodes (i.e., ordinary double points). Such a curve is said to be nodal if it has only nodes as singularities. Salmon proposed the following problem: Describe the situation of nodes of an irreducible nodal curve ([4, Art. 45], [2, pp. 389-393]). Let $n$ denote the degree of a nodal curve and $d$ the number of nodes. The problem is trivial if $n \leq 6$ and $d \leq 8$. The first nontrivial case, $(n, d)=(6,9)$, has been analyzed by Halphen (cf. [2, p. 390]). The case

$$
d \leq \min \{n(n+3) / 6,(n-1)(n-2) / 2\} \quad \text { and }(n, d) \neq(6,9)
$$

was investigated by Arbarello and Cornalba [1, Theorem 3.2]; we give another proof (see Proposition 3(i)). We consider the remaining cases, which are particularly important as they have applications to the moduli variety of curves.

Let $V_{n, d}$ be the variety of irreducible nodal curves of degree $n$ with $d$ nodes. For $n(n+3) / 6 \leq d \leq(n-1)(n-2) / 2$ and $(n, d) \neq(6,9)$, we prove that the map $p_{d}: V_{n, d} \rightarrow \operatorname{Sym}^{d}\left(\mathbf{P}^{2}\right)$, which sends a curve to the set of its nodes, is a birational morphism onto its image (Theorem, Part (i)) and give a rough description of the image (Corollary) and of the generic curve of $V_{n, d}$. In fact, we prove our results for the subvariety $V_{n, d}^{\prime} \subseteq V_{n, d}$ of those nodal curves which can be degenerated into a sum of $n$ lines in general position $\left(V_{n, d}^{\prime}\right.$ is irreducible by $[\mathbf{5}, \S 11])$. We then apply a recent result of Harris to the effect that $V_{n, d}=V_{n, d}^{\prime}[3]$.

2. Zero-dimensional schemes. Let $\mathrm{Hilb}^{e}$ be the Hilbert scheme of zerodimensional subschemes of degree $e$ in $\mathbf{P}^{2}$. One can stratify Hilb ${ }^{e}: Y, Z \in$ Hilb $^{e}$ belong to the same stratum iff $h^{0}\left(\mathbf{P}^{2}, I_{Y}(l)\right)=h^{0}\left(\mathbf{P}^{2}, I_{Z}(l)\right)$ for all $l$. Let $D^{e}$ denote the dense stratum. It is easy to show that $D^{e}$ consists of $m$-regular (in the sense of Castelnuovo) schemes not lying on curves of degree $m-2$, where $m=\min \{i \in \mathbf{Z} \mid e \leq i(i+1) / 2\}$. We denote by $\stackrel{\circ}{D}^{e}$ the subset of $D^{e}$ consisting of schemes of the form $\sum_{i=1}^{e} P_{i}$, where $P_{i} \neq P_{j}$ for $i \neq j$ and $\sum_{k=1}^{d} P_{i_{k}} \in D^{d}$ for every $\left\{i_{1}, \ldots, i_{d}\right\} \subseteq\{1, \ldots, e\}$.

3. Main results. We need four propositions; they are of independent interest.

PROPOSITION 1. Let $d \leq(n-1)(n-2) / 2$. If a reduced curve of degree $n$ with $d$ assigned singular points $P_{1}, \ldots, P_{d}$ is not a specialization of an irreducible curve with $d$ assigned nodes, then $\sum_{i=1}^{d} P_{i} \notin D^{d}$.

Received by the editors February 8, 1987.

1980 Mathematics Subject Classification (1985 Revision). Primary 14H10, 14H45. 
To prove Proposition 1, we suppose $\sum_{i=1}^{d} P_{i} \in D^{d}$ and make a reduction to a curve consisting of two smooth components. We then derive a contradiction by the Cayley-Bacharach theorem.

Let now $L=L_{1}+\cdots+L_{n} \in \mathbf{P}^{2}$ be a sum of $n$ general lines. Set $\left\{P_{1}\right\}=$ $L_{1} \cap L_{2},\left\{P_{2}, P_{3}\right\}=\left\{\left(L_{1}+L_{2}\right) \cap L_{3}\right\} \backslash\left\{P_{1}\right\},\left\{P_{4}, P_{5}, P_{6}\right\}=\left\{\left(L_{1}+L_{2}+\right.\right.$ $\left.\left.L_{3}\right) \cap L_{4}\right\} \backslash\left\{P_{1}, P_{2}, P_{3}\right\}$, etc. Then $\left\{P_{1}, \ldots, P_{d}\right\} \in D^{d}$ for $d \leq n(n-1) / 2$. By Severi $[5, \S 11]$, for $d \leq(n-1)(n-2) / 2, L$ with the assigned nodes $P_{1}, \ldots, P_{d}$ is a specialization of a curve of $V_{n, d}^{\prime}$, and we can prove

PROPOSITION 2. The scheme consisting of $d$ nodes of a general curve of $V_{n, d}^{\prime}$ is a point of $\stackrel{\circ}{D}^{d}$.

In Proposition 3 below, we estimate the dimensions of some families of nonreduced curves. Let $f(x, y, z)=\sum a_{i j k} x^{i} y^{j} z^{k}$ be the homogeneous polynomial of degree $n$ with generic coefficients. We consider the following system of $3 d$ equations in $a$ 's,

$$
f_{x}^{\prime}\left(x_{1}, y_{1}, z_{1}\right)=0, \quad f_{y}^{\prime}\left(x_{1}, y_{1}, z_{1}\right)=0, \quad \ldots, \quad f_{z}^{\prime}\left(x_{d}, y_{d}, z_{d}\right)=0
$$

where $\left(x_{1}: y_{1}: z_{1} ; \ldots ; x_{d}: y_{d}: z_{d}\right) \in\left(\mathbf{P}^{2}\right)^{d}$. Let $M^{d} \subset\left(\mathbf{P}^{2}\right)^{d}$ be the closed subscheme where the system has nontrivial solutions. We have two natural maps Hilb $\stackrel{\phi_{d}}{\longrightarrow} \operatorname{Sym}^{d}\left(\mathbf{P}^{2}\right) \stackrel{\sigma_{d}}{\longrightarrow}\left(\mathbf{P}^{2}\right)^{d}$.

PROPOSITION 3. We assume $[n(n+3) / 6] \leq d \leq(n-1)(n-2) / 2$ and $(n, d) \neq(6,9)$.

(i) Let $K \subset M^{d}$ be an irreducible component and $\left(Q_{1} ; \ldots ; Q_{d}\right) \in K a$ general point. If $d \geq n(n+3) / 6$ and $\sigma_{d}(K) \cap \phi_{d}\left(\stackrel{\circ}{D}^{d}\right) \neq \varnothing$, then a curve of degree $n$ having singularities at $Q_{1}, \ldots, Q_{d}$ is an irreducible nodal curve with $d$ nodes and $\operatorname{dim} K=\operatorname{dim} V_{n, d}$. If $d=[n(n+3) / 6]$, then there exists an irreducible nodal curve with $d$ nodes in general position.

(ii) Let $C \in V_{n, d}^{\prime}$ be a general curve and $P_{1}, \ldots, P_{d}$ its nodes. If $l$ is the degree of a nonreduced curve of minimal degree having singularities at $P_{1}, \ldots, P_{d}$, then $l>n$ unless $(n, d)=(8,14)$.

We also need a generalization of a theorem of Arbarello-Cornalba and Zariski (cf. [6, Theorem 2]).

PROPOSITION 4. Let $A$ be an irreducible analytic family of curves of degree $n$ with $d$ assigned singular points whose general curve, say $B$, is reduced and has $q$ singular points $P_{1}, \ldots, P_{e}, \ldots, P_{d}, \ldots, P_{q}(e \leq d \leq q)$. We asusme $P_{1}, \ldots, P_{d}$ are the assigned singularities, $P_{1}, \ldots, P_{e}$ are nodes, and $P_{e+1}, \ldots, P_{d}$ are not nodes. We also assume:

(i) there exists a curve $C$ of degree $n$ with singularities at $P_{1}, \ldots, P_{d}$, and $C$ and $B$ have no common components,

(ii) $\operatorname{dim} A \geq \operatorname{dim} V_{n, d}-\min \{d-e, n+1\}$. Then $\operatorname{dim} \mathcal{A}=\operatorname{dim} V_{n, d}-d+e$, $q=d$, and $P_{e+1}, \ldots, P_{d}$ are cusps. Furthermore, if $B$ is irreducible, we can drop condition (i) and replace (ii) by the condition:

$$
\operatorname{dim} A \geq \operatorname{dim} V_{n, d}-\min \{d-e, 3(n-1)\} .
$$


THEOREM. (i) If $n(n+3) / 6 \leq d \leq(n-1)(n-2) / 2$ and $(n, d) \neq(6,9)$, then $p_{d}: V_{n, d}^{\prime} \rightarrow \operatorname{Sym}^{d}\left(\mathbf{P}^{2}\right)$ is a birational morphism of $V_{n, d}^{\prime}$ onto its image.

(ii) If $d \leq \min \{n(n+3) / 6,(n-1)(n-2) / 2\}$ and $(n, d) \neq(6,9)$, then for general $P_{1}, \ldots, P_{d} \in \mathbf{P}^{2}$, there exists a curve in $V_{n, d}^{\prime}$ having nodes at $P_{1}, \ldots, P_{d}$.

We prove both assertions simultaneously, first assuming that $[n(n+3) / 6] \leq$ $d \leq(n-1)(n-2) / 2$ and $n \geq 7$. Choose an irreducible component $K \subset M^{d}$ such that $\sigma_{d}^{-1}\left(\overline{p_{d}\left(V_{n, d}^{\prime}\right)}\right) \subseteq K$ and $\operatorname{dim} K=\min \left\{\operatorname{dim} V_{n, d}^{\prime}, 2 d\right\}$. For this $K$, one can find a complete irreducible system $W$ of nodal curves of degree $n$ with $d$ nodes such that $\overline{p_{d}(W)}=\sigma_{d}(K)$. We then show that $\operatorname{dim} W \cap V_{n, d}^{\prime} \geq$ $\operatorname{dim} V_{n, d}^{\prime}-n-1$, and this allows us to deduce from Proposition 4 that $V_{n, d}^{\prime}=W$. The theorem then follows.

We observe that Part (ii) of Theorem also follows from [1, Theorem 3.2] together with [3]. From now on we assume $n(n+3) / 6 \leq d \leq(n-1)(n-2) / 2$ and $(n, d) \neq(6,9)$. Combining our results with the theorem of Harris [3] $\left(V_{n, d}=V_{n, d}^{\prime}\right)$, we obtain

COROllaRY. $\overline{p_{d}\left(V_{n, d}\right)}=\overline{\sigma_{d}\left(M^{d}\right) \cap \phi_{d}\left(\stackrel{\circ}{D}^{d}\right)}$, and for $n(n+3) / 6 \leq t \leq$ $d$, any $t$ nodes of a general curve $C \in V_{n, d}$ determine the location of the remaining nodes of $C$.

REMARK. We can solve (*) on the open subset of $M^{d} \cap \sigma_{d}^{-1}\left(\phi_{d}\left(\stackrel{\circ}{D}^{d}\right)\right)$ where the system has unique solutions, and we obtain the equation of the generic curve of $V_{n, d}$.

\section{REFERENCES}

1. E. Arbarello and M. Cornalba, Footnotes to a paper of Beniamino Segre, Math. Ann. 256 (1981), 341-362.

2. J. L. Coolidge, A treatise on algebraic plane curves, Oxford Univ. Press, Oxford, 1931.

3. J. Harris, On the Severi problem, Invent. Math. 84 (1986), 445-461.

4. G. Salmon, A treatise on the higher plane curves, (2nd ed.), Hodges, Forster, and Co., Dublin, 1873.

5. F. Severi, Vorlesungen über algebraische Geometrie, Anhang F, Teubner, Leipzig, 1921.

6. O. Zariski, Algebraic systems of plane curves, Amer. J. Math. 104 (1982), 209-226.

Department of Mathematics, University of TORONTO, TORONTO, ONTARIO, CANADA M5S 1A1

Current address: Department of Mathematics and Statistics, Queen's University, Kingston, Ontario, Canada K7L 3N6 